\title{
STUDIES ON THE LEVELS OF I7 HYDROXY-CORTICOIDS IN 24-HOUR SPECIMENS OF URINE FROM FIVE QUADRI- PLEGIC PATIENTS AND TWO PARAPLEGIC PATIENTS ADMITTED TO THE ROYAL NORTH SHORE HOSPITAL, SYDNEY
}

\author{
By J. M. F. Grant and J. D. Yeo \\ Paraplegic Unit, Royal North Shore Hospital, Sydney, Australia
}

Introduction. It has been observed clinically that a number of quadriplegic patients suffer from intermittent periods of 'vacancy', sometimes leading to stupor with associated hyponatraemia and eosinophilia. Hypotension not necessarily related to posture has been noted as part of this syndrome. We consider that there may be an isolated deficiency of ACTH production from the anterior pituitary gland as a cause of symptoms in this small group of patients, and therefore an initial study of the levels of 17 hydroxy-corticoids in their urine specimens was carried out. In two of these patients a more detailed study of the pituitary-adrenal axis was made.

Clinical Methods and Material. The following investigations were considered essential in the investigation of this syndrome:

I. Estimation of 17 hydroxy-corticoids in 24 hours specimens of urine.

2. ACTH stimulation test.

3. Metapirone stimulation test.

4. Hypoglycaemia and plasma cortisol levels including investigation of the presence of a normal 'diurnal' variation.

5. Creatinine clearance test and/or blood urea.

6. Intravenous pyelogram.

7. Electro-encephalography.

8. Skull X-ray.

9. Thyroid function tests.

I0. Growth hormone levels.

II. Random blood estimations.

The clinical details of two quadriplegic patients are now presented:

Patient I. G. T., female, aged I8 years, who was involved in a car accident on 23 August 1963 when she suffered an anterior dislocation of $\mathrm{C}_{4}$ vertebra with spinal cord compression at that level and a resultant complete tetraplegia below $\mathrm{C}_{4}$ spinal level.

She was first discharged in December 1964 in good general health, with a balanced automatic neurogenic bladder, but severe generalised somatic spasm. She was readmitted in July I966 with recurrent episodes of disorientation and 'vacancy'. She appeared to be suffering from a significant urinary tract infection, a marked anaemia (9·7 g. per cent.), thrombocytopaemia (40,000 c.b. mg.) due apparently to the toxic effects of sulphonamide therapy. Following treatment for her urinary tract infection and transfusion for the moderately severe anaemia, her symptoms subsided. During this admission serum electrolytes were estimated and found to be normal except for the serum sodium which was I32 mEq./L (normal I35-I 55). After discharge she had symptoms related to autonomic hyper-reflexia. Studies on a 24-hour collection of urine showed normal levels 
of I 7 ketosteroids ( $8.6 \mathrm{mg}$., normal 3-I2) and I7 hydroxy-corticoids (I6.3, normal values 3-I2). An E.E.G. showed moderate diffuse abnormality during her in-patient state.

On I6 September I966 she was again re-admitted for the investigation of disturbance of concentration, vomiting, fever and menorrhagia. She was noted to have ecchymoses on the tongue, haemoglobin $7 \cdot 8 \mathrm{~g}$. per cent., blood pressure on admission $85 / 65$. Physical examination revealed the presence of a pansystolic murmer in the mitral region, and a diagnosis of bacterial endocarditis was was made. Investigations included blood urea $75 \mathrm{mg}$. per cent. (sudden rise); serum calcium 9:4 mg. per cent., serum proteins normal; platelets 90,000/c.mm., white cell count 9200/c.mm., of normal distribution. No L.E. cells seen. E.S.R. 57/103; urine-significant infection of Enterobacter cloacae and Enterococcus. Blood cultures were negative. Repeated random blood sugars-within normal limits.

Direct Coombs test negative. Clot retraction inhibition test using Tetracycline, Gantrisin and Negram revealed no inhibiting of retraction in the presence of these drugs. I7 ketosteroids $2.9 \mathrm{mg}$./day, I7 OH corticoids $2.9 \mathrm{mg}$./day. Metapirone stimulation test four fold increase. E.C.G. was normal. E.E.G. normal.

Impression. This girl was considered to be suffering from a gram-negative septicaemia with urinary tract infection and secondary thrombocytopaenia. Drugs tested did not appear involved. Evidence of bacterial endocarditis disappeared within one week and the patient was discharged well, free of significant symptoms. It was thought that the low adrenal output appeared to be due to lack of response factors of anterior pituitary via the hypothalamic pathways, despite evidence of severe infection.

Haemoglobin and platelet count had returned to normal levels, at discharge on 13 October 1966.

Thyroid function test carried out on 24 October I966 ( $I^{131}$ uptake 6) appeared within normal limits and P.B.I. ${ }^{131}$ was within the normal range. This patient was later found to have a normal diurnal variation of plasma cortisol levels, and a normal increase of these levels was produced by significant Hypoglycaemia ( $20 \mathrm{mg}$. per cent.). Growth hormone levels have also been estimated and lie within normal limits ( $\mathrm{I} \mathrm{mcg}$. per mille) (normal $0 \cdot 7 \mathrm{I}-8$ ). There has been no clinical evidence of disturbance of gonadotrophins but these hormones have not been estimated quantitatively. This patient has remained well over the last six months without any further specific treatment.

Patient 2. D. C., male, aged 2 I years. This patient was admitted to the hospital on 9 May I965 following a motor-cycle injury in which he suffered a fracture dislocation of C5 and C6 vertebrae with compression of the spinal cord at C6 level and a resultant incomplete spastic quadriplegia below this spinal level.

Nine weeks after admission he had progressed satisfactorily so that he was allowed to sit up using a cervical collar. One week later he suffered from recurrent grand mal epilepsy which was controlled only with great difficulty. E.E.G. studies revealed that he was suffering from centrencephalic epilepsy which did not recur under treatment with Dilantin and Prominal. It was noted that he had a low serum sodium (134 mEq./litre) and eosinophilia (I200/c.mm. 27 August I965) and I7 hydroxycorticoids 6.9 mg./day and I 7 ketosteroids $6.4 \mathrm{mg}$./day. There was no skin pigmentation and he had an adequate response to ACTH stimulation test, on the fifth day.

The Metapirone test also revealed an adequate increase in 17 Hydroxy-corticoids present in urine.

On repeating the 17 hydroxy-corticoids levels in urine it was found that in his 'resting' state the level was only $3.8 \mathrm{mg}$ per 24 hours (normal level 6-I 5). Following his discharge he suffered from recurrent urinary tract infection despite antibiotic control and it was therefore decided to give him a supplementary dose of Betamethasone, $0.5 \mathrm{mg}$. daily to aid his resistance to infection. Troublesome symptoms of autonomic hyperreflexia were controlled with the use of Serpasil, $0.25 \mathrm{mg}$. t.d.s. Renal function tests including the I.V.P. have remained within normal limits. Skull X-ray N.A.D. 
He was discharged on 6 July 1966 and has remained well since that date. He has since been investigated by studying the pattern of his plasma cortisol levels and a normal diurnal variation has been found. With the production of significant hypoglycaemia (25 per cent.) a significant rise in the level of plasma cortisol was produced. A recent E.E.G. was repeated-N.A.D. without anticonvulsants. Thyroid function tests-no abnormality detected; growth hormone levels $0.4 \mathrm{mg}$./mille (normal levels $0 \cdot 7-8 \cdot 0$ ).

Patient 3. J. L., male, aged 2 I years, with a complete spinal cord injury below $\mathrm{C}_{5}$ level, following a motor vehicle accident on 8 February 1967.

On 26 April 1967-I7 hydroxy-corticoids, 5.5 mg. (normal 9.5-19). Subsequent investigations revealed normal thyroid function tests, normal diurnal variation of plasma cortisol levels.

Patient 4. D. L., male, aged I 5. In complete quadriplegia below C6 spinal level, with continued recovery, following motor vehicle accident on 27 November 1966.

On 9 January 1967 hydroxy-corticoid levels were $4.4 \mathrm{mg}$. per day (normal in practice 9-19). On 23 January 1967 metapirone tests produced a response from $4.7 \mathrm{mg}$. to $6.5 \mathrm{mg}$. per day. Thyroid function tests were found to be within normal limits and he also had a normal diurnal variation of plasma cortisol.

Patient 5. G. B., male, aged is years, who is completely quadriplegic below $\mathrm{C}_{5}$ spinal level following an injury on I6 November I966 (some sparing of sensation).

On 9 January 1967 I7 hydroxy-corticoid level was $3.3 \mathrm{mg}$. (normal 9-I9). On 20 January 1967 metapirone stimulation test produced a rise from $4.7 \mathrm{mg}$. to $7.3 \mathrm{mg}$. per 24 hours. This boy had a normal diurnal variation of plasma cortisol.

In the above three patients renal function tests were normal (including I.V.P.'s) and E.E.G.'s were within normal limits. The estimation of 17 ketosteroids were also found to be within acceptable normal limits on all these patients.

Patients 6 and 7. The remaining two patients in this series were female, with complete paraplegia at TI2 and T8 Spinal levels respectively, and these were found to have normal levels ('resting') of I 7 hydroxy-corticoids and I 7 ketosteroid levels in 24-hour specimens of urine.

Stimulation tests have not been, as yet, carried out on these patients.

\section{DISCUSSION}

Methods of investigation and clinical details of the above patients are presented as an indication of our interest to further investigate what appears to be a recurrent clinical entity in some quadriplegics. The pathogenesis of this low basal output state of the adrenal cortex is not at present clear to us but we would suggest the following possibilities:

(a) Neurogenic, due to the deficient afferent input to the hypothalamus and to the anterior pituitary and subsequent deficient function of the pituitary-adrenal axis.

(b) A vascular disturbance:

I. As for example in the Sheehan syndrome, where hypotension results in thrombotic involvement of the essential portal venous channels.

2. Ischaemia, whether acute or recurrent in the region supplied by the vertebro-basilar arterial system.

In conclusion. We consider these findings worthy of the collection of further clinical material and data, and would be most interested to hear of the experiences of other Centres who have studied this syndrome in the quadriplegic patient. 